Trauma Berufskrankh 2004 - 6 [Suppl 2] : S258-S261 DOI 10.1007/s10039-003-0777-1

Online publiziert: 9.0 ktober 2003

(c) Springer-Verlag 2003

E. Mayr • Abteilung für Unfallchirurgie, Klinikum der Universität Regensburg

\title{
Winkelstabile Plattenosteosynthese der distalen Tibia
}

Stichinzisionen nach entsprechender Reposition der Fraktur an den Knochen angelagert.

Diese Technik der „durchgeschobenen Platte“ birgt allerdings die Gefahr des primären sowie des sekundären Repositionsverlusts.

Primärer Repositionsverlust. Der primäre Repositionsverlust (• Abb. 1) entsteht dadurch, dass beim Besetzen der Plattenlöcher mit entsprechenden Schrauben einzelne Fragmente an die Platte herangezogen werden. Da diese Platten aber in der durchgeschobenen Technik nicht exakt konturiert werden können, ist es möglich, dass die Fragmente in eine Fehlstellung gezogen werden.

Sekundärer Repositionsverlust. Für den sekundären Repositionsverlust (• Abb. 2) ist weniger die ungenügende Konturierung der Platte als vielmehr die Stabilität des Gesamtkonstrukts verantwortlich. Insbesondere bei vorliegenden Trümmerzonen kann es hier wegen der fehlenden interfragmentären Abstützung zum sekundären Abweichen der beiden Hauptfragmente kommen.

\section{Implantatvorstellung}

Sowohl dem primären als auch dem sekundären Repositionsverlust kann effektiv mit einem winkelstabilen Osteosynthesesystem entgegengewirkt werden. Dies führte zur Entwicklung winkelstabiler Plattenfixateure.

\section{LCP-Pilonplatte}

Die LCP-Pilonplatte (• Abb. 3) hat die Form eines Ankers, wobei der Ankerschaft mit klassischen LCP-Löchern ausgearbeitet ist. Diese Löcher können sowohl mit einer winkelstabilen Schraube als auch mit einer herkömmlichen Kortikalis- bzw. Spongiosaschraube auch unter Verwirklichung des dynamischen Plattenprinzips, wie es von der LCDCP her bekannt ist, besetzt werden. Im Bereich der beiden queren Schenkel sind ausschließlich Löcher zur Aufnahme von winkelstabilen Verriegelungsschrauben vorgesehen. Am Übergang vom Schaft zu den beiden queren Schenkeln können sowohl herkömmliche Kleinfragmentschrauben als auch winkelstabile Verriegelungsschrauben in den Rundlöchern verankert werden.

\section{Distale LCP-Tibiaplatte}

Die distale Tibia-LCP (• Abb. 4) hat ebenfalls einen Schaft, welcher mit typischen LCP-Löchern besetzt ist. Am distalen Plattenende findet sich eine löffelartige Verbreiterung, welche Rundlöcher aufweist, die für die Aufnahme von winkelstabilen Verriegelungsschrauben gedacht sind.

\section{Technik der Plattenimplantation}

Aufgrund ihrer Gestalt eignet sich die LCP-Pilonplatte nicht als „durchgeschobene Platte“. Die beiden distalen queren Schenkel müssen der jeweiligen anatomischen Situation angeformt werden. Zwischen den einzelnen Plattenlöchern kann die Platte mit einem Seitenschnei- 


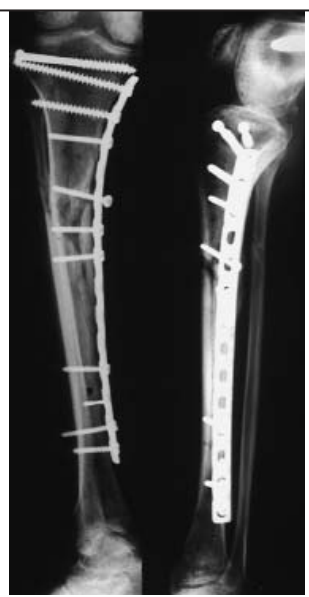

Abb. $1<$ Primärer Repositionsverlust von $60^{\circ}$ Außenrotation und $15^{\circ}$ Varus

der in der Länge jeweils angepasst werden. Das Biegen der beiden queren Schenkel erfolgt entweder mit 2 Flachzangen oder mit 2 in die Plattenlöcher eingeschraubten Bohrhülsen des LCP-Systems (- Abb. 5). Letztere Variante hat den Vorteil, dass die Gewindelöcher während des Biegevorgangs vor Verformung geschützt sind. Deshalb sollte diese Technik bevorzugt werden.

Die distale LCP-Tibiaplatte eignet sich dagegen hervorragend für eine minimalinvasive Technik, bei der die Platte über eine kleine Inzision auf Höhe des Innenknöchels nach proximal unter den Weichteilen durchgeschoben wird (• Abb. 6).

Da bei dieser Technik jedoch keine Möglichkeit zur Konturierung des Plattenschafts gegeben ist, muss bei der Verwendung von Kleinfragmentschrauben im Schaftbereich die Möglichkeit eines primären Repositionsverlusts berücksichtigt werden. Deshalb ist es sinnvoll, entweder alle Schrauben winkelstabil zu besetzen oder aber die Platte zunächst mit 1 oder 2 konventionellen Kleinfragmentschrauben am proximalen Hauptfragment $\mathrm{zu}$ fixieren und erst in einem 2 . Schritt die Metaphyse mit winkelstabilen Schrauben zu stabilisieren.

\section{Klinischer Nutzen}

\section{Geometrie und ihre Folgen}

Die beiden hier vorgestellten winkelstabilen Plattenfixateure bieten eine sinnvolle Bereicherung des unfallchirurgischen Implantatspektrums. Um die Implantate Gewinn bringend einsetzen zu können, muss

Trauma Berufskrankh 2004 - 6 [Suppl 2] : S258-S261

DOI 10.1007/s10039-003-0777-1

C) Springer-Verlag 2003

E. Mayr

\section{Winkelstabile Plattenosteosynthese der distalen Tibia}

\section{Zusammenfassung}

Bereits mit herkömmlichen Osteosyntheseplatten wurde versucht, eine biologische Osteosynthese ohne Denudierung der Fragmente zu erzielen - mit der Gefahr des primären oder sekundären Repositionsverlusts. In den letzten Jahren wurden winkelstabile Plattenfixateure entwickelt, darunter 2 für die distale Tibia (LCP-Pilonplatte, distale LCP-Tibiaplatte). Sie stellen eine Bereicherung des traumatologischen Implantatspektrums dar. Bei sehr weit distal gelegenen Tibia- und bei Pilon-tibial-Frakturen konkurrieren diese mit der herkömmlichen Plattenosteosynthese. Sowohl die geometrischen Daten der winkelstabilen Verriegelungsschrauben als auch die Tatsache, dass sie mit ihrem Kopfgewinde fest in der Platte verankert sind, machen deutlich, dass sie nicht als Zugschrauben geeignet sind, sondern in der Platte

\section{Fixed-angle plating of the distal tibia}

\section{Abstract}

Attempts were made with conventional internal fixation plates to achieve biological internal fixation without denudation of the fragments - with the inherent danger of a primary or secondary realignment loss. In recent years fixed-angle plate fixators have been developed, including two for the distal tibia (LCP pilon plate, distal LCP tibial plate). They have extended and improved the range of implants available for the treatment of traumatic fractures, and their use now rivals the implementation of conventional plate fixation for the treatment of tibial fractures at far distal sites and of pilon tibial factures. Both the geometric specification of the fixed-angle bolts and the fact that the thread at their head fixes them firmly in the plate make it clear that they are not suitable winkelstabil verankerte Bolzen darstellen. Dies muss bei der Planung der Osteosynthese berücksichtigt werden. Meist müssen zusätzlich Kompressionsschrauben eingesetzt werden. Da die neuen Implantate und Schrauben wesentlich teurer sind als herkömmliche Systeme, muss der Einsatz jeder einzelnen Schraube sowohl aus ökonomischer als auch aus biomechanischer Sicht hinterfragt werden. Beim kombinierten Einsatz von Verriegelungsschrauben und Kleinfragmentschrauben ist der mögliche Repositionsverlust zu bedenken.

\section{Schlüsselwörter}

Biologische Osteosynthese · Winkelstabiler Plattenfixateur · LCP-Pilonplatte · Distale LCP-Tibiaplatte $\cdot$ Kosten · Repositionsverlust for use as tension screws but are stably fixed in the plate at fixed angles. This must be taken into account when the internal fixation is planned. It is usually necessary to use compression screws in addition. Since the new implants and screws cost considerably more than conventional systems, the use of each and every screw must be both economically and biomechanically justified. Attention must be paid to the possibility of realignment loss when locking screws and small-fragment screws are used together.

\section{Keywords}

Biological internal fixation - Fixed-angle plate fixator - LCP pilon plate - LCP plate for distal tibia . Cost $\cdot$ Realignment loss 


\section{Plattenosteosynthesen}

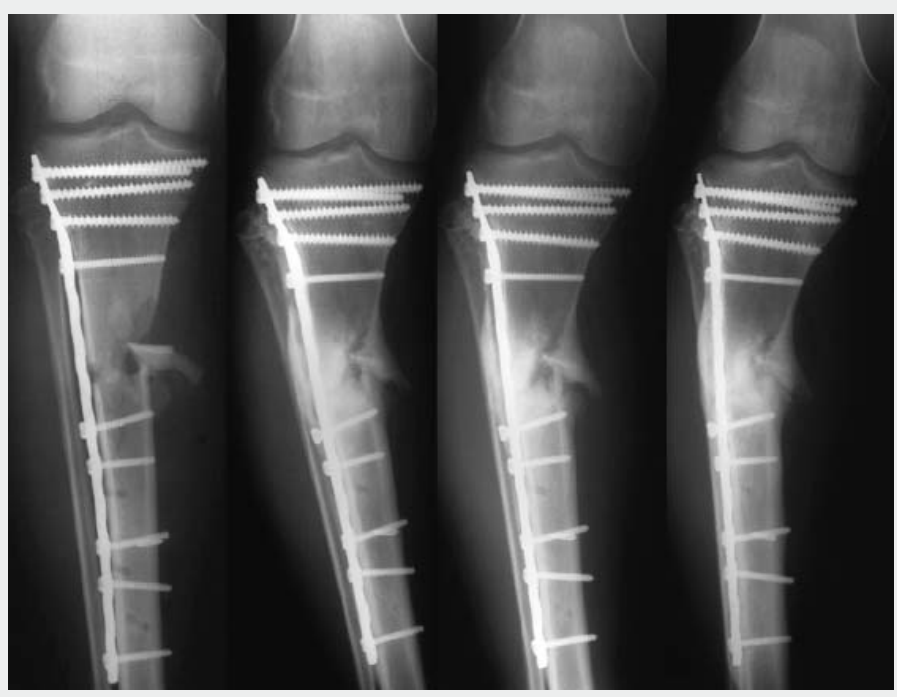

Abb. $2 \Delta$ Sekundärer Repositionsverlust im Lauf von 5 Monaten postoperativ

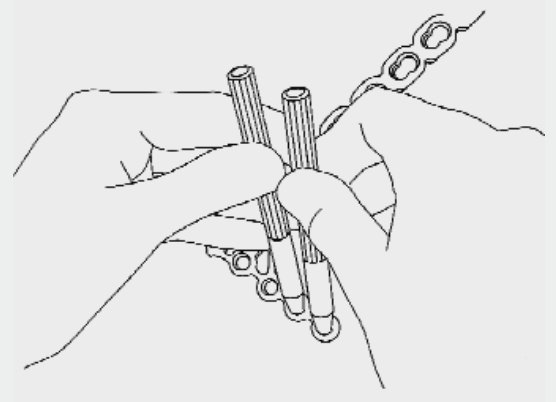

Abb. $5 \Delta$ Biegevorgang der Pilon-LCP

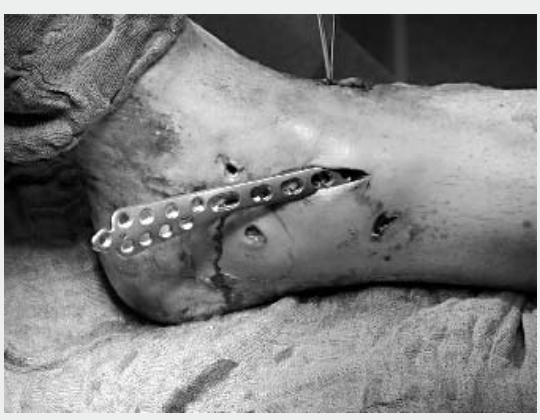

Abb. $6 \Delta$ Eingeschobene Technik der distalen Tibia-LCP

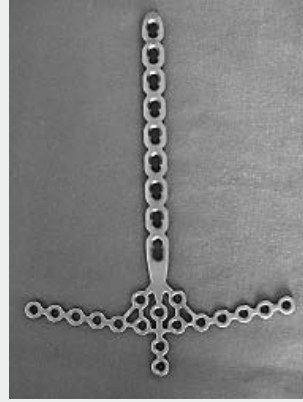

Abb. $3 \Delta$ LCP-Pilonplatte

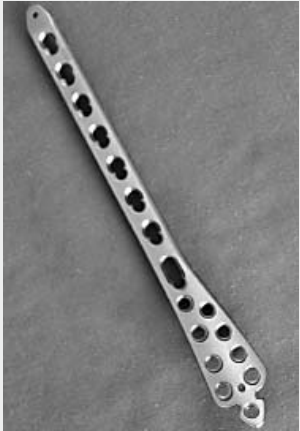

Abb. $4 \Delta$ Distale Tibia-LCP

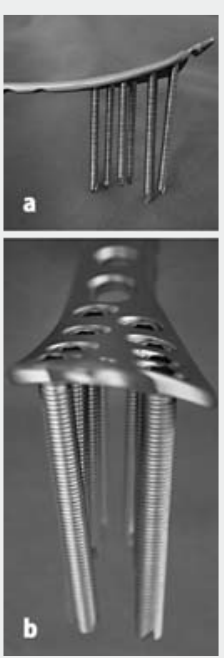

Abb. $7<$ Anordnung der Verriegelungsschrauben bei der distalen Tibia-LCP man sich allerdings über die Implantatgeometrie im Klaren sein.

Im Vergleich zu den herkömmlichen Kleinfragmentschrauben weisen die winkelstabilen Verriegelungsschrauben erhebliche Unterschiede in ihrer Geometrie auf (- Tabelle 1): So ist der Kerndurchmesser der Verriegelungsschraube mit 2,9 mm größer als der der Kleinfragmentkortikalis- $(2,4 \mathrm{~mm})$ und v. a. der der Kleinfragmentspongiosaschraube (1,9 mm). Sowohl die Gewindesteigung als auch noch ausgeprägter - die Gewindetiefe sind bei der Verriegelungsschraube am kleinsten. Winkelstabile Verriegelungsschrauben stehen nur als Vollgewindeschrauben zur Verfügung.

Sowohl die geometrischen Daten der winkelstabilen Verriegelungsschrauben als auch die Tatsache, dass sie mit ihrem Kopfgewinde fest in der Platte verankert sind, wodurch das Eindrehen limitiert ist, machen deutlich, dass die Verriegelungsschrauben nicht als Zugschrauben geeignet sind. Vielmehr handelt es sich hier um in der Platte winkelstabil verankerte Bolzen. Dieser Tatsache muss bei der Planung und Durchführung der Osteosynthese unbedingt Rechnung getragen werden. In den allermeisten Fällen wird man deshalb auf isolierte Kompressionsschrauben nicht verzichten können. Allerdings besteht durch die winkelstabile Ausbildung aller Schrauben an den distalen Enden der beiden hier vorgestellten winkelstabilen Platten die Gefahr einer Interaktion der Verriegelungsschrauben mit isolierten Zugschrauben und auch Frakturspalten.

Von Seiten der Industrie werden die winkelstabilen Platten insbesondere bei osteoporotischen Knochen als vorteilhaft bezeichnet. Dies ist jedoch bislang mit keiner experimentellen Untersuchung oder klinischen Studie bewiesen und gründet lediglich auf der theoretischen Überlegung, dass es im Fall eines sekundären Repositionsverlusts bei der konventionellen Technik zum Schraubenausriss und im Fall des Plattenfixateurs zum Durchschneiden der Schrauben durch Knochen kommt, was mit einer angeblich höheren Reibung einhergeht. Allerdings bleibt bei dieser theoretischen Überlegung die Schraubengeometrie mit den erheblichen Unterschieden in Gewindesteigung und Gewindetiefe zwischen Kleinfragmentschrauben und Verriegelungsschrauben unberücksichtigt. Darüber hinaus muss auch die Frage gestellt werden, wie viel Winkelstabilität ein osteoporotischer Knochen verkraften kann. Es wäre in diesem Zusammenhang durchaus vorstellbar, dass ein winkelstabiler Plattenfixateur zu rigide ist und somit dem Implantatversagen und damit dem sekundären Repositionsverlust Vorschub leistet. 
Tabelle 1

Implantatgeometrie von Kleinfragmentkortikalisschraube (1. Reihe), Kleinfragmentspongiosaschraube

(2. Reihe) und Verriegelungsschraube (3. Reihe)

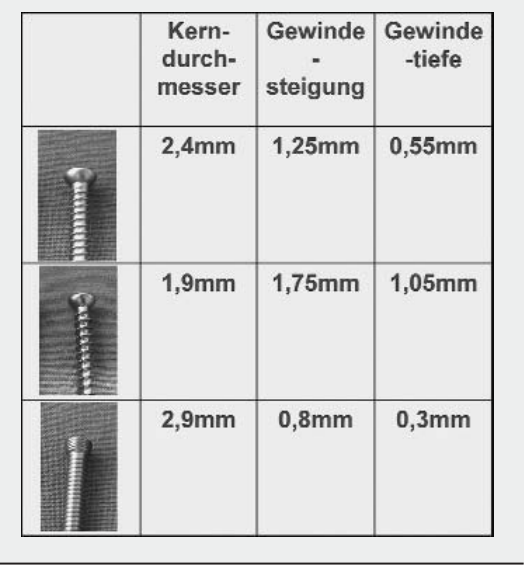

Tabelle 2

\section{Vergleich der Implantatkosten}

Implantat

Kosten

[EURO]

8-Loch-LCDCP, $4,5 \mathrm{~mm}$ schmal

82,52

40-mm-Großfragmentschraube

10,24

Kleeblattplatte

182,64

40-mm-Kleinfragmentschraube $\quad 8,36$

Distale Tibiaplatte (LCP) $\quad 392,68$

Pilonplatte (LCP) $\quad 366,79$

40-mm-Verriegelungsschraube $\quad 30,69$

Betrachtet man eine distale Tibia LCP mit einliegenden Schrauben im metaphysären Bereich, fällt auf, dass diese lediglich in 2 unterschiedlichen Ebenen angeordnet sind ( $\bullet$ Abb. 7). Durch eine in allen 3 Ebenen divergierende Anordnung der Schrauben hätte man die Stabilität der Plattenverankerung hier entscheidend verbessern können.

\section{Kosten und ihre Folgen}

Ein Vergleich der Kosten (• Tabelle 2) zeigt, dass die distale Tibia-LCP etwa 4,5-mal so teuer ist wie die konkurrierende 8-Loch-LCDCP, 4,5 $\mathrm{mm}$ schmal, und dass die Pilon-LCP etwa doppelt so teuer ist wie die konkurrierende Kleinfragmentkleeblattplatte. Bei den Schrauben besteht ein Kostenfaktor von etwa 3,5 zwischen konventionellen AO-Schrauben und den Verriegelungsschrauben. Dies zeigt, dass man sich auch aus ökonomischen Gründen den Einsatz jeder einzelnen Verriegelungsschraube genau überlegen muss und auch, ob der Nutzen die Kosten wirklich rechtfertigt. Prinzipiell sollte diese Überlegung immer angestellt werden, wenn es um die Frage geht, ob man nun ein winkelstabiles oder ein herkömmliches Plattensystem verwenden will. Bei konsequenter Durchführung dieser Überlegungen ist durch die detaillierte Auseinandersetzung mit den zu behandelnden Frakturen allerdings ein erheblicher Schulungs- und Ausbildungseffekt zu erwarten.

\section{Fazit für die Praxis}

Sicherlich handelt es sich bei der Entwicklung der distalen Tibia-LCP und der Pilon-LCP um

eine notwendige und sinnvolle Bereicherung des traumatologischen Implantatspektrums. Beim Umgang mit diesen Implantaten ist allerdings erhebliche Disziplin gefordert. Der Einsatz jeder einzelnen winkelstabilen Schraube sollte sowohl aus ökonomischer als auch aus biomechanischer Sicht hinterfragt werden. Bei der Mischung von Verriegelungsschrauben und Kleinfragmentschrauben muss man sich über die Mechanik jeder einzelnen Schraube im Klaren sein, da hier die theoretische Möglichkeit des primären Repositionsverlusts besteht.

\section{Korrespondierender Autor Prof.Dr. E. Mayr}

Abteilung für Unfallchirurgie, Klinikum der Universität Regensburg, Franz-Josef-Strauss-Allee 11,93042 Regensburg, E-Mail: edgar.mayr@klinik.uni-r.de 